\title{
Subaperture stitching interferometry based on digital holography
}

\author{
Feng Pan*, Xiaoyun Lu, Bin Dong, Xichao Ma and Wen Xiao \\ ${ }^{I}$ Key Laboratory of Precision Opto-mechatronics Technology, \\ School of Instrument Science and Optoelectronics Engineering, Beihang University, Beijing 100191, China \\ *panfeng@buaa.edu.cn \\ Ph:+86-01-82339736
}

\begin{abstract}
A novel subaperture stitching interferometry based on digital holography is developed to measure the deformation of spherical surfaces. The subaperture measurement is performed by off-axis digital holography on single exposure. Then, the subaperture phase maps are obtained by digital holographic reconstruction, in which the phase aberration caused by position errors of each subaperture measurement is effectively compensated by the method of numerical parametric lens. After that, the full aperture phase map is retrieved by a subaperture stitching algorithm, in which the relative alignment errors of adjacent subapertures are eliminated with an iterative process of stitching optimization. The experiments demonstrate the feasibility and effectiveness of the proposed interferometry, which provides a rapid and robust way to measure spherical surfaces with high resolution and precision. A practical example is given to demonstrate the performance of this method. The stitching result shows good agreement with the full-aperture result.
\end{abstract}

Key words: Digital holography; Subaperture stitching; Interferometry; Surface measurements; Optical testing.

\section{Introduction}

In the application of high precision spherical elements, such as sphere optics, sphere bearing, gyro rotator, calibration spheres, etc., the surface quality is very important to the performance of the instruments. Optical interferometry is an established tool for the measurement of spherical surfaces. However, all interferometers are limited in the maximum object size they can measure and in the spatial details they can resolve. The limited slope sampling capability of commercial standard interferometers presents the technical and cost challenges to test spherical surfaces with high numerical aperture (NA), especially such as the whole surfaces of a sphere or a hemisphere. The subaperture stitching interferometry (SSI) has been proved to be an effective technique to test spheres with high NA [1-4]. The basic idea of SSI is to measure many subapertures of the tested surface and to synthesize a full aperture map from them. Generally, a standard interferometer is used to acquire the subaperture phase maps individually by rotating the tested sphere. And then, the full aperture phase map can be obtained by stitching these subaperture results via a certain stitching algorithm.

The significant application of SSI was the subaperture stitching interferometer workstation produced by QED Technologies. This workstation is capable of extending the effective aperture, accuracy, resolution, and dynamic range of a standard interferometer, and can automatically carry out high quality subaperture stitching of flat, spherical, and aspherical surfaces. It combined a six-axis precision stage system, a commercial Fizeau interferometer, and specially developed software to orchestrate measurement design, motion control, data acquisition, reconstruction of the fullaperture map [5-8]. CSIRO's Australian Centre for Precision Optics has devised and built a subaperture stitching interferometer for production of a high precision, high numerical aperture spherical mirror. In the measuring system, the subaperture stitching was utilized by a commercial phase-shift interferometer and a specialized swivel mount $[9,10]$. With the aspherical mirrors used more and more widely in optical systems, the subaperture stitching interferometry are proposed for testing large and deep rotational symmetric aspheric surfaces [11-15]. In fact, the subaperture stitching algorithm plays a vital role in the SSI. The earliest stitching methods were able to test large optical flats by fitting polynomials to non-overlapping subapertures [16]. The improved stitching algorithms attempted to achieve consistent synthesis by mathematically minimizing the mismatch among the overlapping areas of multiple subapertures [17]. A significant improvement was realized by simultaneously fitting rigid-body alignment terms among multiple overlapping subapertures [18-25]. Recently, Shanyong Chen et al. proposed a self-calibrated subaperture stitching algorithm for hyper-hemispheres tests, based on the alternating optimization technique and the successive linearization method [26-28]. To our best knowledge, the existing SSI utilizes a commercial phase-shifting interferometry to acquire the subaperture phase maps. The phase shifting process is not only consumes time, but sensitive to the environment during measurement process. Although there are several techniques for instantaneously obtaining all phase-shifted interferograms on a single camera for phase-shifting interferometry [1, 29-31]. Some additional optical elements can be added to the testing optical path and complex algorithms can be used to process the phase or compensate the errors. In addition, Yichun Chen et al. have proposed a vibration modulated subaperture stitching interferometry by adopting the random phase shifting caused by the stage mechanical vibrations [32,33]. It brings a large amount of data and computational problems. On the other hand, it has been noted that the spherical geometry leads to phase-shifting errors, which cause errors in the figure map for testing a high NA spherical surface $[34,35]$.

Digital holography (DH) is one of the state of the art digital wavefront reconstruction techniques [36]. Compared with the classical holography, the hologram is recorded by an electronic sensor array (e.g. Charged Coupled Device, CCD, or Complementary Metal Oxide Semiconductor, CMOS), and the holographic reconstruction is numerically achieved with a computer. On the basis of a single off-axis hologram, it can produce not only an amplitude image, but also a quantitative 
phase contrast image [37-40]. It means that the topography measurements on tested surface with optical interferometric precision and without vibration isolation. Moreover, due to the inherent advantages of DH such as numerical focusing [41-44], numerical aberration correcting [38, 40, 45, 46], synthetic aperture imaging [47-51] and super-resolution imaging [52-55], the morphology measurement can be achieved with a large field of view and high resolution. Therefore, it has the potential for addressing rapid measurement of surface quality in realistic workshop conditions (e.g., under mechanical vibration, air turbulence, and so on) with high resolution and precision. Consequently, a novel subaperture stitching interferometry based on DH is proposed to measure deformation of spherical surfaces and the enhanced robustness of the method is discussed in this paper.

\section{Methods}

\subsection{Measurement system}

The sketch map of the experimental setup is shown in Fig.1, and the layout is presented in Fig.2. The basic architecture is that of a Mach-Zehnder interferometer. A linear He-Ne laser $(632.8 \mathrm{~nm}, 5 \mathrm{~mW})$ is used as the light source. The combination of the polarizing beam splitter (PBS) and the half-wave plate $(\lambda / 2)$ is used for the adjustment of the intensity ratio of the beams in the reference arm and the object arm. In the reference arm a half-wave plate $(\lambda / 2)$ is introduced to obtain parallel polarization states in both arms at the exit of the interferometer. The beam expanders $\left(\mathrm{BE}_{1}, \mathrm{BE}_{2}\right)$, including pinholes for spatial filtering, are introduced to produce plane waves. For reflection testing, a custom-made complex lens (CL) with high NA is inserted between the BS and the tested sphere. This lens produces a converging spherical wave with PV less than $1 / 10 \lambda(\lambda=633 \mathrm{~nm})$ and the half-angle of the light cone of $35^{\circ}$. Based on null configuration, the center of curvature of curvature of the tested surface is positioned to coincide with the focus of the spherical wave emanating from the CL, as shown in Fig.1. Therefore, the reflected wavefront, called the object wave, provides information about the figure and irregularity of the surface under test. The object wave $\boldsymbol{O}$ and the reference wave $\boldsymbol{R}$ are recombined by the BS and interfere at the exit of the interferometer, where a black and white CMOS camera (the resolution of $2048 \times 2048$ pixels, the pixel size of $3.45 \times 3.45 \mu \mathrm{m}$ ) records the hologram intensity which is then transmitted to a computer via USB interface. In order to create off-axis holograms, the orientation of the mirror (M2) which reflects the reference wave is adjusted so that the reference wave reaches the CMOS with a tilt angle, while the object wave propagates perpendicularly to the hologram plane.

In this arrangement, each subaperture needs to be nulled uniquely for the tested sphere by placing the center of curvature of the part at the focus of the illuminating spherical wave. Therefore, a five degree of freedom stage is designed to adjust the tested sphere. It comprises three linear axes (X, Y and Z) and two rotary axes (A and B), as shown in Fig.2 (b). The tested sphere is stuck to a rod linking to the rotary axes of A, which coincides with the symmetrical axis of the sphere. The rotary axes of B is also yawing from the central of curvature of the tested surface and peripheral with the rotary axes of $\mathrm{A}$. That is, the intersection of the two rotation axes coincides with the center of curvature of the tested surface. A three linear translator is used to adjust the center of curvature of the tested surface, and ensures its position coincident with the focus of the converging wave. In fact, the accuracy of the stage is not sufficient to place the positon of each subaperture within a specified alignment tolerance. As a result, the re-alignment has to be done until all subaperture holograms have been obtained. The residual alignment errors of each subaperture are corrected in the data processing, as illustrated in the next section.

\subsection{Data process}

\subsubsection{Subaperture reconstruction}

As previously mentioned, a platform with five degrees of freedom is needed to implement the adjustment and bring each subaperture into null adjustment. However, due to the existence of manual operations, it is impossible to obtain precise position and pose parameters of the tested sphere. Furthermore, this system is complex and without vibration isolation, making it very sensitive to environmental vibration. The effects of small random positioning system errors may cause the phase aberrations for each subaperture. These errors include the piston, tilt, and power, and are different in each subaperture. Therefore, the numerical aberration correction is applied to compensate the phase aberrations of the subapertures prior to performing the stitching operation. The relative alignment errors of adjacent subapertures will be overcome by the optimization process of the stitching algorithm. This method combines the use of a manual adjustor and of the numerical aberration correction. Fig. 3 illustrates the whole process of holographic reconstruction and subaperture stitching procedures for the proposed SSI.

Once all of the subaperture holograms have been obtained, numerical reconstruction is performed to retrieve subaperture phase maps. The subaperture holograms are given as

$$
h_{i}=|R|^{2}+\left|O_{i}\right|^{2}+R^{*} O_{i}+R O_{i}^{*} \mid i=1, \ldots, s
$$

where $\boldsymbol{R}$ is the reference wave, $\boldsymbol{O}$ is the object wave, $|\cdot|$ is the modulus operation, $*$ is the complex conjugate operation, and $s$ is the number of the subapertures. The so-called hologram apodization is carried out to avoid diffraction ripples on the reconstructed wavefront, which is caused by the finite nature of the camera chip, by multiplying the hologram with a two dimensional window function, such as a cubic spline interpolation function [56]. The processed holograms are expressed as $\left\{\bar{h}_{i} \mid i=1, \ldots, s\right\}$. In order to isolate the high-intensity zero-order term and the twin image term in an off- 
axis hologram, a spatial filter can be applied in the Fourier spatial frequency domain, by computing the Fourier transform of the hologram [57]. The filtered holograms are given as $\left\{\bar{h}_{i}^{\text {Filter }}=R^{*} O_{i} \mid i=1, \ldots, s\right\}$. In fact, they correspond to the reference modulated complex object wavefront in the hologram plane.

In order to compensate the phase aberrations caused by residual alignment errors, the numerical aberration correction is achieved in the hologram plane. If not considering the shape errors of the tested surface, the phase aberration can be expressed as

$$
\Phi_{i}=\varphi_{i}^{\mathrm{DC}}+\varphi_{i}^{\mathrm{Xtilt}}+\varphi_{i}^{\mathrm{Ytilt}}+\varphi_{i}^{\text {Power }} \mid i=1, \ldots, s
$$

where $\varphi^{\mathrm{DC}}$ is the piston aberration, $\varphi^{\mathrm{Xtilt}}$ and $\varphi^{\text {Ytilt }}$ are the two directional tilt aberrations, and $\varphi^{\text {Power }}$ is the defocus aberration. Specifically, the first-order tilt aberrations $\varphi^{\text {Xtilt }}$ and $\varphi^{\text {Ytilt }}$ associate with the holographic off-axis angle and a minor deviation of the center of curvature of the tested surface from focus of the illuminating spherical wave in the horizontal (X) and vertical (Y) directions, respectively. The second-order defocus aberration $\varphi^{\text {Power }}$ associates with a minor deviation of the center of curvature of the tested surface from the focus of the illumination spherical wave in the optical axis direction $(\mathrm{Z})$. The constant bias aberration $\varphi^{\mathrm{DC}}$ is created between different subaperture measurements. The phase aberrations can be individually compensated by using the method of Numerical Parametric Lens (NPL) [38, 40]. The NPL is a two dimensional polynomial phase, which is indeed specifically designed against the above-mentioned aberrations. For generating the NPL, a two dimensional polynomial fit is fulfilled according to the phase map of $\left\{\arg \left[\bar{h}_{i}^{\text {Filter }}\right] \mid i=1, \ldots, s\right\}$, where $\arg []$ is the imaginary part operation. Once the phase mask $\left\{\phi_{i}^{F i t} \mid i=1, \ldots, s\right\}$ has been fitted by using a quadratic polynomial function, the object wave on the hologram plane can be given as $\left\{\bar{h}_{i}^{\text {Filter }} \cdot \exp \left[-i \phi_{i}^{\text {Fit }}\right] \mid i=1, \ldots, s\right\}$ without the aberration caused by residue position errors.

The subaperture phase maps are retrieved by using convolution propagation algorithm [58], and expressed as

$$
\Psi_{i}=\mathrm{F}^{-1}\left\{\mathrm{~F}\left[\bar{h}_{i}^{\text {Filter }} \cdot \exp \left(-i \phi_{i}^{F i t}\right)\right] \cdot \exp \left[-j \pi \lambda d\left(v_{x}^{2}+v_{y}^{2}\right)\right]\right\} \mid i=1, \ldots, s
$$

where $\Phi$ is Fourier transform operator, $\Phi^{-1}$ is inverse Fourier transform operator, $\lambda$ is the optical wavelength, $d$ is the reconstruction distance, $v_{\mathrm{x}}=k /(N \Delta x)$ and $v_{\mathrm{y}}=l /(M \Delta y)$ are the spatial frequencies in the $x$ and $y$ direction, respectively, $k$ and $l$ are integer numbers, $N$ and $M$ are the number of pixels along both directions, $\Delta x$ and $\Delta y$ are the pixel sizes of the camera in both directions. Finally, the subaperture phase maps are expressed as $\left\{p_{i}=\arg \left[\Psi_{i}\right] \mid i=1, \ldots, s\right\}$.

\subsubsection{Subaperture stitching}

Afterward, a stitching algorithm is utilized to synthesize a full aperture phase map by estimating and correcting relative alignment errors of adjacent subapertures [26]. First, based on initial position parameters, the subaperture phase maps are synthesized into a full aperture phase map in a general coordinate frame. The phase values of subapertures is given as $\left\{p_{j, i} \mid j=1, \ldots, N_{i} ; i=1, \ldots, s\right\}$ in local coordinate frames. Then the full aperture phase map is given as in global coordinate frame as [28]

$$
\mathrm{P}_{i}=g_{i}^{-1} p_{j, i} \mid j=1, \ldots, N_{i} ; i=1, \ldots, s
$$

where $\mathrm{g}=\left\{g_{i} \in \mathrm{SE}(3) \mid i=1, \ldots, s\right\}$ are configurations of the $i$ th subapertures. It is given as $\mathrm{SE}(3)=\left\{\exp \left(\sum_{t=1}^{6} m_{t} \xi_{t}\right)\right\}$ by using a special Euclidean group, where $\xi=\left[\begin{array}{cc}\hat{\omega} & v \\ 0 & 0\end{array}\right]$ is referred to as a twist and $\xi=(v \omega)^{T}$ as the twist coordinates, $\xi_{t} \in \mathrm{R}^{6}$ is a unit vector whose $i$ th element is 1 and otherwise 0 . Owing to uncertainties of the swivel stage, the initialized phase map suffers from stitching errors and presents non-consistent. Second, the overlapping correspondences are exactly recognized and the deviations among the subapertures are computed. To find overlapping points between adjacent subapertures, such as the $k$ th and the $i$ th subapertures, all points in the two subapertures are projected onto the nominal surface, yielding corresponding projections $\left\{x_{j, k}\right\}$ and $\left\{x_{j, i}\right\}$. If a point in $\left\{x_{j, k}\right\}$ lies in the area of $\left\{x_{j, i}\right\}$, the point is defined as lying in the overlapping region. Meanwhile, a signed distance of a point in the $i$ th subaperture to the nominal surface is expressed as

$$
\operatorname{dis}_{j, i}=\left\langle g_{i}^{-1} p_{j, i}-x_{j, i}, n_{j, i}\right\rangle
$$

where $n_{j, i}$ is the unit normal vector of the nominal surface at a point of $x_{j, i}$. A signed deviation of the $k$ th and $i$ th subapertures is expressed as

$$
\operatorname{dev}_{j, i}=\left\langle g_{k}^{-1} p_{j o, k}-x_{j o, k}, n_{j o, k}\right\rangle-\left\langle g_{i}^{-1} p_{j o, i}-x_{j o, i}, n_{j o, i}\right\rangle
$$


where the subscript $j o \in\left\{1, \ldots, N_{o}\right\}$ indicates the points in the overlapping region between the pair of subapertures, $N_{\mathrm{o}}$ is the total number of overlapping point pairs in the $k$ th and $i$ th subapertures. Third, an objective function is defined as $f=\mu_{1} \mathrm{RMS}^{2}+\mu_{2} \mathrm{RMS}_{\mathrm{o}}^{2}$, where $\mathrm{RMS}$ and $\mathrm{RMS}_{\mathrm{o}}$ are the root mean squares of signed distances to the nominal surface and signed deviations of two adjacent subapertures, respectively, and represented as

$$
\begin{aligned}
\mathrm{RMS}^{2} & =\sum_{i=1}^{s} \sum_{j=1}^{N_{i}}[\operatorname{dis}(j, i)]^{2} / \sum_{i=1}^{s} N_{i} \\
\mathrm{RMS}_{\mathrm{o}}^{2} & =\sum_{i=1}^{s-1} \sum_{k=i+1}^{s} \sum_{j o=1}^{N_{o}}[\operatorname{dev}(j, i)]^{2} / N_{o}
\end{aligned}
$$

where $\mu_{1}$ and $\mu_{2}$ are positive weights satisfying $\mu_{1}+\mu_{2}=1$. Fourth, for finding the optimal configuration $g$, the objective function $f$ is minimized by an iteration calculation to update the configuration as $g_{i}^{l+1}=g_{i}^{i}\left(I+\sum_{t=1}^{r} m_{t, i} \xi\right), i=1, \ldots S$, where $I$ is a $4 \times 4$ identity matrix. That is, the overlapping and deviation calculations in the second step are alternately solved until the object function $f$ converges be within an acceptable tolerance. Finally, the full aperture phase map is obtained with according to the optimized configuration parameters of $\boldsymbol{g}$.

\section{Results and analyses}

The experiments are demonstrated to verify the effectiveness of the proposed SSI. A convex spherical lens with the radius of curvature of $30 \mathrm{~mm}$ and an effective aperture of $25.4 \mathrm{~mm}$ is measured, and the result is compared with that obtained by a commercial Fizeau interferometer. In order to cover the full aperture of the tested surface and provide sufficient overlaps, twelve subapertures are measured individually, including eleven peripheral subapertures and a central one, as shown in Fig. 4. As mentioned above, the tested lens is aligned so that its center of curvature is confocal with the illuminating spherical wave. The central subaperture is located by coinciding the optical axis with the axis of symmetry. The first peripheral subaperture is located by rotating the stage against the axis (B) so that the angle between the symmetric axis and the optical axis is about $32^{\circ}$. Then, the remaining subapertures are located by rotating the stage against the symmetrical axis (A). A subaperture hologram is shown in Fig.5 (a), where an enlarged region marked by the white dashed rectangle is shown in Fig.5 (b). By using holographic numerical reconstruction with the reconstruction distance $d$ of $31.15 \mathrm{~cm}$, the results are shown in Fig.5 (c) and (d) with later resolution of $10 \mu \mathrm{m}$. Fig.5 (c) presents the phase map with only compensating the tilt aberration caused by off-axis configure, where the tilt and defocus aberrations caused by residual position error is clearly visible. After compensating the residual aberrations, the phase map frees from aberrations is shown in Fig.5 (d). Based on the reconstructed phase maps, a full aperture phase map is obtained by stitching them together with each initial position and pose parameters. The result is shown in Fig.6 with the PV of 0.295 $\lambda$ and RMS of $0.064 \lambda$, where the stitching errors and overlapping inconsistency are obvious. By using the subaperture stitching algorithm, the result is represented in Fig. 7 with PV of $0.247 \lambda$ and RMS of $0.051 \lambda$, where a region marked by the rectangle is enlarged with $350 \times 350$ pixels in Fig.7 (a), and Fig.7 (b) gives a globe demonstration. To confirm the stitching result, a cross-test is carried out by a Zygo interferometer. The result (no stitching) is shown in Fig. 8 with the PV of $0.224 \lambda$ and RMS of $0.054 \lambda$. It is noticed that the figure error from the proposed SSI agrees quite well with that from the Zygo interferometer. Although there are slightly differences in marginal areas, the averaging of the large amounts of subaperture data makes the stitching result smoother.

Furthermore, the proposed SSI is applied to measure a hemisphere bearing with a radius of curvature of $12 \mathrm{~mm}$, as shown in Fig. 2 (a). Nine subapertures are designed to finish the full aperture testing, as shown in Fig.9. The stitching result is shown in Fig. 10 with the PV of $0.521 \lambda$ and RMS of $0.056 \lambda$, where the region marked by the rectangle gives the morphological details on the surface.

\section{Conclusion}

In this paper, a subaperture stitching interferometry based on digital holography is proposed for the measurement of spherical elements. Each subaperture measurement is achieved by recording an off-axis digital hologram on a single exposure. Moreover, the phase aberrations of each subaperture caused by the position errors can be effectively compensated by numerical reconstruction processing. Afterward, the full aperture phase map is retrieved by synthesizing the subaperture phase maps with a subaperture stitching algorithm, which minimizes the alignment errors of adjacent subapertures. The experimental results show the stitched figure error agrees well with the no stitching result from a commercial interferometer. The proposed interferometry has a good performance for testing spherical surface. Additionally, the interferometry provides a rapid and robust way for testing spherical elements with high resolution and accuracy in realistic workshop conditions.

\section{Acknowledgments}

This work is supported by the National Science Foundation of China (No. 61177006). 


\section{Reference}

[1] D. Malacara, Optical Shop Testing, 3rd ed (Wiley 2007).

[2] J. E. Negro, "Subaperture optical system testing," Appl. Opt. 23(12), 1921-1930 (1984).

[3] P. Murphy, G. Forbes, J. Fleig, P. Dumas, and M. Tricard, "Stitching interferometry: A flexible solution for surface metrology," Opt. Photonics News 14(5), 38-43 (2003).

[4] S. Chen, S. Li, Y. Dai, L. Ding, and S. Zeng, "Experimental study on subaperture testing with iterative stitching algorithm," Opt. Express 16(7), 4760-4765 (2008).

[5] J. Fleig, P. Dumas, P. E. Murphy, G. W. Forbes, “An automated subaperture stitching interferometer workstation for spherical and aspherical surfaces," Proc. SPIE 5188, 296-307, (2003).

[6] M. Tricard, P. Dumas, and G. Forbes, "Sub-aperture approaches for asphere polishing and metrology," Proc. SPIE 5638, 284-299 (2005).

[7] M. Tricard, A. Kulawiec, M. Bauer, G. DeVries, J. Fleig, G. Forbes, D. Miladinovich, and P. Murphy, "Subaperture stitching interferometry of high-departure aspheres by incorporating a variable optical null," Manu. Tech., 59, 547-505 (2010).

[8] P. E. Murphy, D. Stephenson, A. E.W. Jones, and G. W. Forbes, "Design of systems involving easily measurable aspheres," Proc. SPIE 8884, 1D-1-10 (2013).

[9] J. Burke, K. Green, W. Stuart, E. Puhanic, A. Leistner, and B. Oreb, "Fabrication and testing of a high-precision concave spherical mirror," Proc. SPIE 7064, 7064E-1-10 (2008).

[10] M. Sjodahl, B. F. Oreb, "Stitching interferometric measurement data for inspection of large optical components," Opt. Eng., 41(2), 403-408 (2002).

[11] Xi Hou, Fan Wu, Li Yang, Shibin Wu, and Qiang Chen, "Full-aperture wavefront reconstruction from annular subaperture interferometric data by use of Zernike annular polynomials and a matrix method for testing large aspheric surfaces," Appl. Opt. 45, 3442-3455 (2006).

[12] Lei Zhang, Chao Tian, Dong Liu, Tu Shi, Yongying Yang, Hanshuo Wu, and Yibing Shen, "Non-null annular subaperture stitching interferometry for steep aspheric measurement," Appl. Opt. 53, 5755-5762 (2014).

[13] Wen Y, Cheng H, "Measurement for off-axis aspheric mirror using off-axis annular subaperture stitching interferometry: theory and applications," Opt. Eng. 54, 014103-1-11 (2014).

[14] Lei Zhang, Dong Liu, Tu Shi, Yongying Yang, Shiyao Chong, Baoliang Ge, Yibing Shen, and Jian Bai, "Aspheric subaperture stitching based on system modeling," Opt. Express 23, 19176-19188 (2015).

[15] Gao F, Jiang Z, Zhao Z, Li B, "Measurement of aspheric surface combining point diffraction interferometry and annular subaperture stitching,". Opt. Eng. 54, 014102-1-5 (2015).

[16] W. Chow and G. Lawrence, "Method for subaperture testing interferogram reduction”, Opt. Lett., 8, 468-470, 1983.

[17] T. W. Stuhlinger, "Subaperture optical testing: experimental verification," Proc. SPIE 656, 118-127 (1986).

[18] M. Otsubo, K. Okada, and J Tsujiuchi, "Measurement of large plane surface shapes by connecting small-aperture interferograms," Opt. Eng. 33, 608-613, (1994).

[19] Michael Bray, "Stitching interferometer for large plano optics using a standard interferometer," Proc. SPIE 3134, 39-50, (1997).

[20] Michael Bray, "Stitching interferometer for large optics: recent developments of a system," Proc. SPIE 3492, $946-$ 956 (1999).

[21] Sjo“dahl M, Oreb BF, "Stitching interferometric measurement data for inspection of large optical components," Opt. Eng. 41, 403-408 (2002).

[22] P. E. Murphy, G. W. Forbes, J. F. Fleig, D. Miladinovic, G. DeVries, and S. O'Donohue, "Recent Advances in Subaperture Stitching Interferometry," in Frontiers in Optics, OSA Technical Digest (CD) ( 2006).

[23] Pengfei Zhang, Hong Zhao, Xiang Zhou, and Jinjun Li, "Sub-aperture stitching interferometry using stereovision positioning technique," Opt. Express 18, 15216-15222 (2010).

[24] Lisong Yan, Xiaokun Wang, Ligong Zheng, Xuefeng Zeng, Haixiang Hu, and Xuejun Zhang, "Experimental study on subaperture testing with iterative triangulation algorithm," Opt. Express 21, 22628-22644 (2013).

[25] Hongru Li, Guoying Feng, Jianfei Sun, Thomas Bourgade, Shouhuan Zhou, Anand Asundi, "Wavefront subaperture stitching with Shack-Hartmann sensor," Proc. SPIE 9524, 2189452 (2015).

[26] S. Chen, W. Liao, Y. Dai and S. Li. "Self-calibrated subaperture stitching test of hyper-hemispheres using latitude and longitude coordinates," Appl. Opt. 51(17), 3817-3825 (2012).

[27] S Chen, S Li, and Y Dai, "Iterative algorithm for subaperture stitching interferometry for general surfaces," J. Opt. Soc. Am. A 22, 1929-1936 (2005).

[28] S. Chen, S. Li, Y. Dai, and Z. Zheng, "Iterative algorithm for subaperture stitching test with spherical interferometers," J. Opt. Soc. Am. A 23, 1219-1226 (2006).

[29] Robert M. Neal and James C. Wyant, "Polarization phase-shifting point-diffraction interferometer," Appl. Opt. 45, 3463-3476 (2006).

[30] Gustavo Rodriguez-Zurita, Noel-Ivan Toto-Arellano, Cruz Meneses-Fabian, and José F. Vázquez-Castillo, "Oneshot phase-shifting interferometry: five, seven, and nine interferograms," Opt. Lett. 33, 2788-2790 (2008).

[31] N. I. Toto-Arellano, D. I. Serrano-García, A. Martínez-García, G. Rodríguez-Zuríta and A. Montes-Pérez, "4D profile of phase objects through the use of a simultaneous phase shifting quasi-common path interferometer", Journal of Optics, Vol. 13, pp 115502-115510, (2011). 
[32] C.W. Liang, H. S. Chang, P. C. Lin, C. C. Lee and Y. C. Chen. "Vibration modulated subaperture stitching interferometry,”Opt. Express 21(15), 18255-18260 (2013).

[33] H. S. Chang, C. W. Liang, P. C. Lin, and Y. C. Chen, "Measurement improvement by high overlapping density subaperture stitching interferometry," Appl. Opt., 53(29), H102-H108 (2014).

[34] K. Creath, P. Hariharan, "Phase-shifting error in interferometric tests with high-numerical-aperture reference surfaces", Appl. Opt., 33(1), 24-25 (1994).

[35] P. D. Groot, "Phase-shift calibration errors in interferometers with spherical Fizeau cavities", Appl. Opt., 34(16), 2856-2863 (1995).

[36] U. Schnars, C. Falldorf, J. Watson and W. Juptner, Digital Holography and Wavefront Sensing, 2nd ed (Springer 2014).

[37] E. Cuche, F. Bevilacqua, and C. Depeursinge, "Digital holography for quantitative phase-contrast imaging," Opt. Lett., 24(5), 291-293 (1999).

[38] P. Ferraro, S. D. Nicola, A. Finizio, G. Coppola, S. Grilli, C. Magro, and G. Pierattini, "Compensation of the inherent wave front curvature in digital holographic coherent microscopy for quantitative phase-contrast imaging," Appl. Opt., 42(11), 1938-1946 (2003).

[39] C. J. Mann, L. Yu, C. M. Lo, and M. K. Kim, "High-resolution quantitative phase-contrast microscopy by digital holography," Opt. Express 13(22), 8693-8698 (2005).

[40] T. Colomb, E. Cuche, F. Charrière, J. Kühn, N. Aspert, F. Montfort, P. Marquet, and C. Depeursinge, “Automatic procedure for aberration compensation in digital holographic microscopy and applications to specimen shape compensation," Appl. Opt., 45(5), 851-863 (2006).

[41] P. Langehanenberg, B. Kemper, D. Dirksen, and G. von Bally, "Autofocusing in digital holographic phase contrast microscopy on pure phase objects for live cell imaging," Appl. Opt. 47, 176-182 (2008).

[42] P. Gao, B. Yao, J. Min, R. Guo, B. Ma, J. Zheng, M. Lei, S. Yan, D. Dan and T. Ye, “Autofocusing of digital holographic microscopy based on off-axis illuminations," Opt. Lett. 37, 3630-3632 (2012).

[43] F. Dubois, A. El Mallahi, J. Dohet-Eraly, and C. Yourassowsky, "Refocus criterion for both phase and amplitude objects in digital holographic microscopy," Opt. Lett. 36, 4286-4289 (2014).

[44] P. Memmolo, M. Paturzo, B. Javidi, P. A. Netti and P. Ferraro, "Refocusing criterion via sparsity measure-ments in digital holography," Opt. Lett. 39, 4719-4722 (2014).

[45] T. Colomb, F. Montfort, J. Kühn, N. Aspert, E. Cuche, A. Marian, F. Charrière, S. Bourquin, P. Marquet, and C. Depeursinge, "Numerical parametric lens for shifting, magnification, and complete aberration compensation in digital holographic microscopy,” J. Opt. Soc. Am. A. 23(12), 3177-3190 (2006).

[46] S. Liu, W. Xiao and F. Pan, "Automatic compensation of phase aberrations in digital holographic microscopy for living cells investigation by using spectral energy analysis," Opt. \& Las. Tech. 57,169-174 (2014).

[47] V. Micó, Z. Zalevsky, P. G. Martínez, and J. García, "Synthetic aperture superresolution with multiple off-axis holograms," J. Opt. Soc. Am. A 23, 3162-3170 (2006).

[48] Lluís Martínez-León and Bahram Javidi, "Synthetic aperture single-exposure on-axis digital holography," Opt. Express 16, 161-169 (2008).

[49] Melania Paturzo and Pietro Ferraro, "Correct self-assembling of spatial frequencies in super-resolution synthetic aperture digital holography," Opt. Lett. 34, 3650-3652 (2009).

[50] G. Coppola, G. Di Caprio, M. Gioffré, R. Puglisi, D. Balduzzi, A. Galli, L. Miccio, M. Paturzo, S. Grilli, A. Finizio, and P. Ferraro, "Digital self-referencing quantitative phase microscopy by wavefront folding in holographic image reconstruction," Opt. Lett. 35, 3390-3392 (2010).

[51] A. Pelagotti, M. Paturzo, M. Locatelli, A. Geltrude, R. Meucci, A. Finizio, and P. Ferraro, "An automatic method for assembling a large synthetic aperture digital hologram," Opt. Express 20, 4830-4839 (2012).

[52] Cheng Liu, Zhigang Liu, Feng Bo, Yong Wang and Jianqiang Zhu, "Super-resolution digital holographic imaging method," Appl. Phys. Lett. 81(17), 3143-3145 (2002).

[53] M. Paturzo, F. Merola, S. Grilli, S. De Nicola, A. Finizio, and P. Ferraro, "Super-resolution in digital holography by a two-dimensional dynamic phase grating," Opt. Express 16, 17107-17118 (2008).

[54] L. Granero, Z. Zalevsky, and V. Micó, "Single-exposure two-dimensional superresolution in digital holography using a vertical cavity surface-emitting laser source array," Opt. Lett. 36, 1149-1151 (2011).

[55] A. Hussain, J. L. Martínez, A. Lizana, and J. Campos, "Super resolution imaging achieved by using on-axis interferometry based on a Spatial Light Modulator," Opt. Express 21, 9615-9623 (2013).

[56] E. Cuche, P. Marquet, and C. Depeursinge, “Aperture apodization using cubic spline interpolation: application in digital holographic microscopy,” Opt. Commun. 182(1-3), 59-69 (2000).

[57] E. Cuche, P. Marquet, and C. Depeursinge, "Spatial filtering for zero-order and twin-image elimination in digital off-axis holography," Appl. Opt. 39(23), 4070-4075 (2000).

[58] T. M. Kreis, "Frequency analysis of digital holography with reconstruction by convolution," Opt. Eng. 41(8), 18291839 (2002).

\section{List of figure captions}

Fig.1 Sketch of experimental setup.

Fig.2 Layout of experimental setup.

Fig.3 Flow chart of subaperture reconstruction and stitching process. 
Fig.4 Sketch of subaperture measurement for a convex spherical lens.

Fig.5 Subaperture measurement by DH, (a) a subaperture hologram, (b) enlarged region of hologram, (c) phase map without position errors compensation, (d) phase map with position errors compensation.

Fig.6 Initial stitching result of convex spherical lens with the PV of $0.295 \lambda$ and RMS of $0.064 \lambda$.

Fig.7 Stitching result of convex spherical lens by the proposed interferometry with PV of $0.247 \lambda$ and RMS of $0.051 \lambda$, (a) the stitching result, (b) a globe show.

Fig.8 Full aperture result of convex spherical lens by Zygo interferometer with the PV of $0.224 \lambda$ and RMS of $0.054 \lambda$. Fig.9 Sketch of the subaperture measurement for a hemisphere bearing.

Fig.10 Stitching result of hemisphere bearing with the PV of $0.521 \lambda$ and RMS of $0.056 \lambda$, (a) the stitching result, (b) a globe show. 


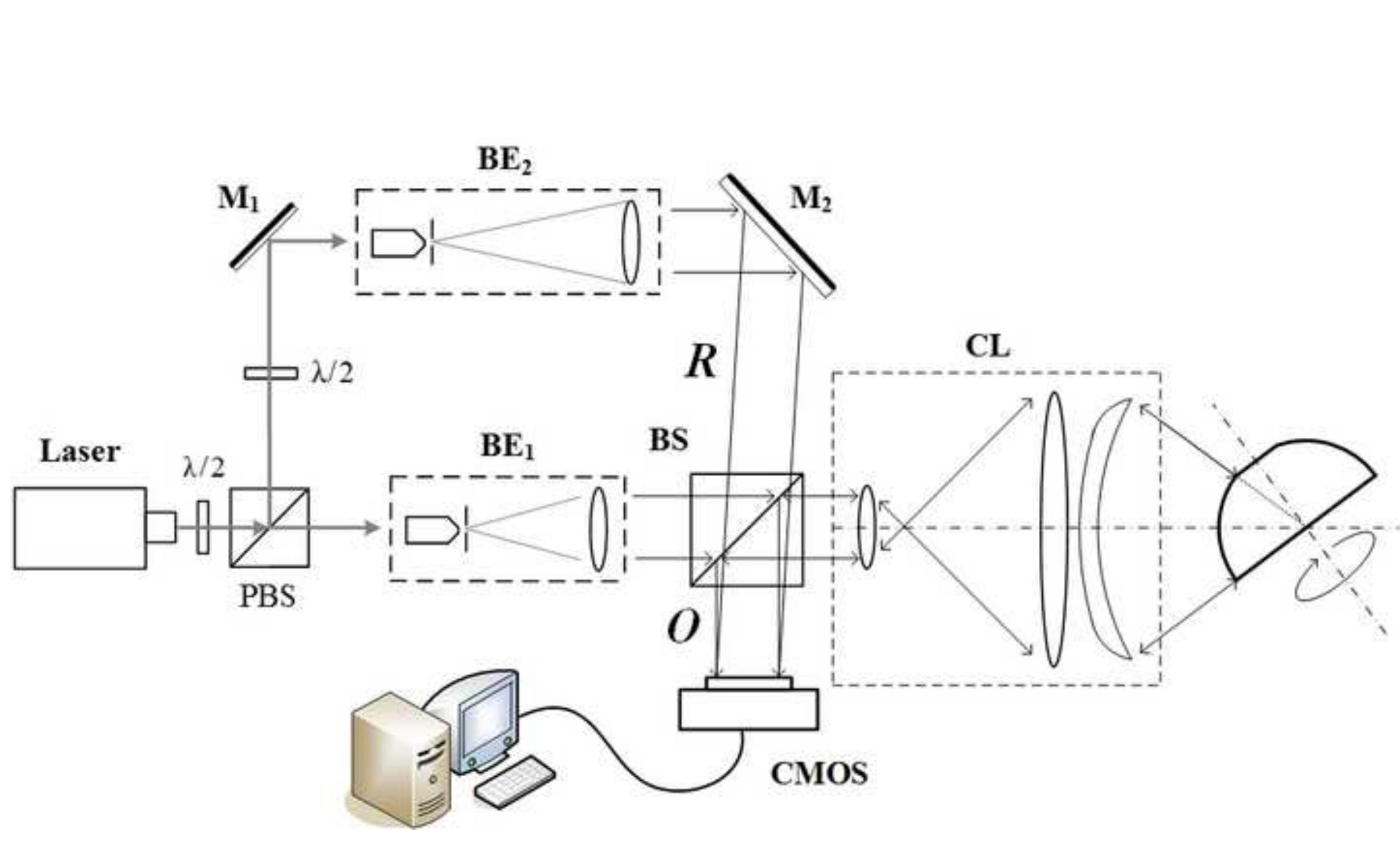



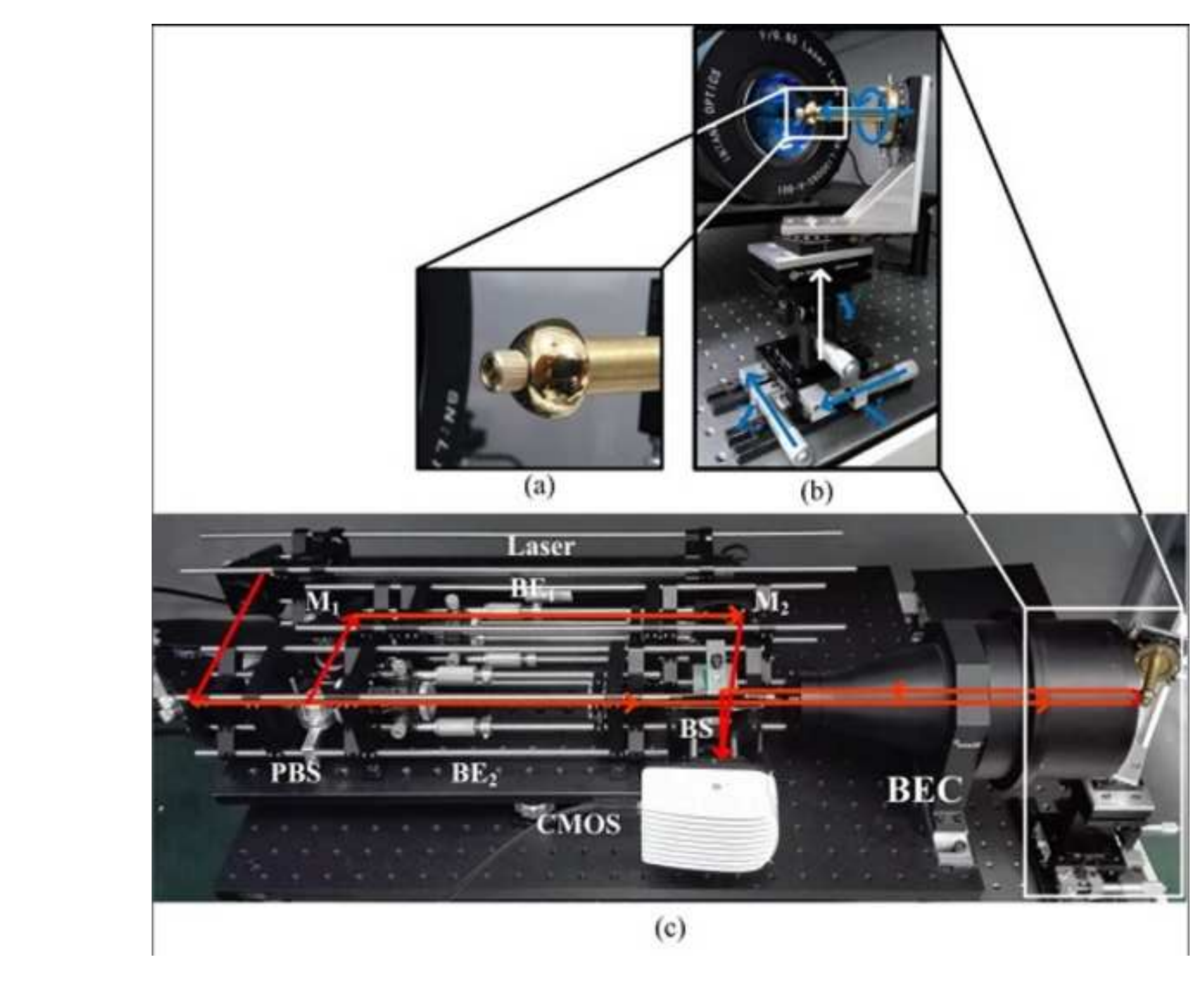


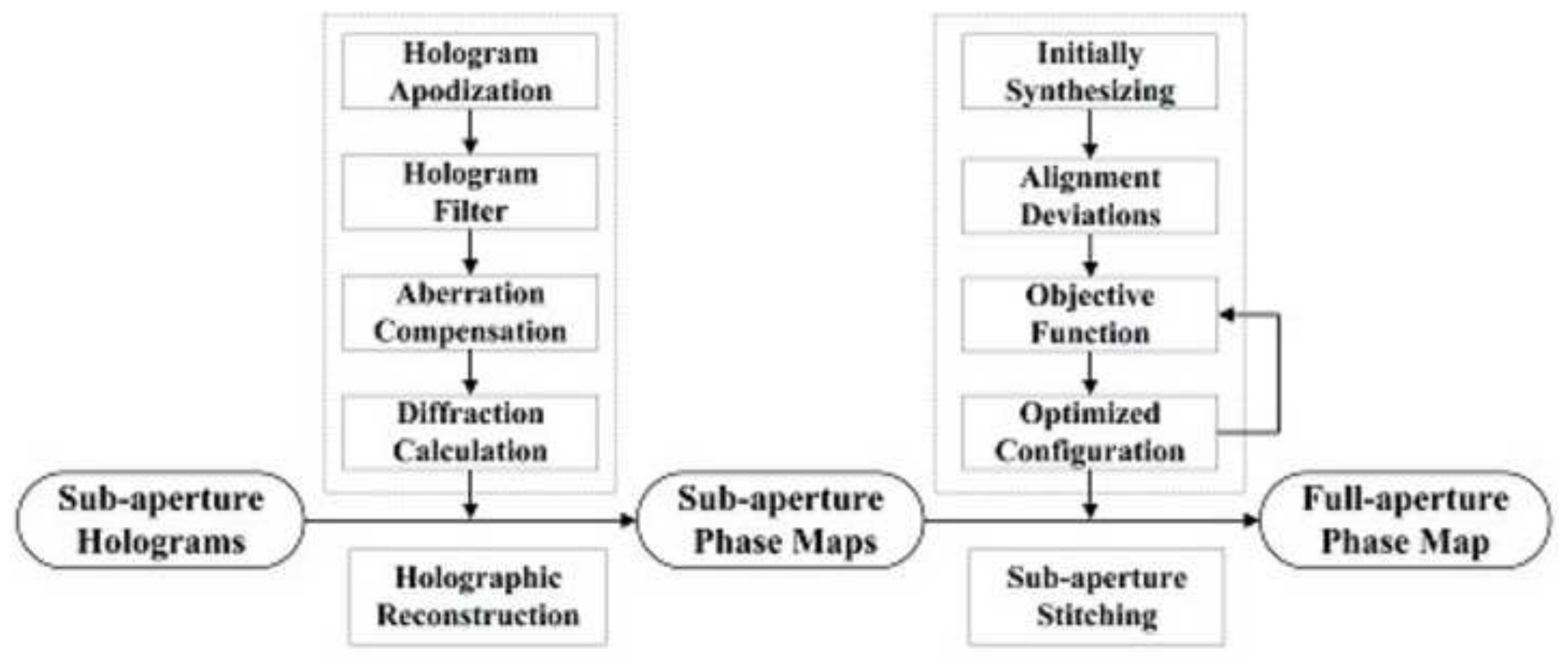




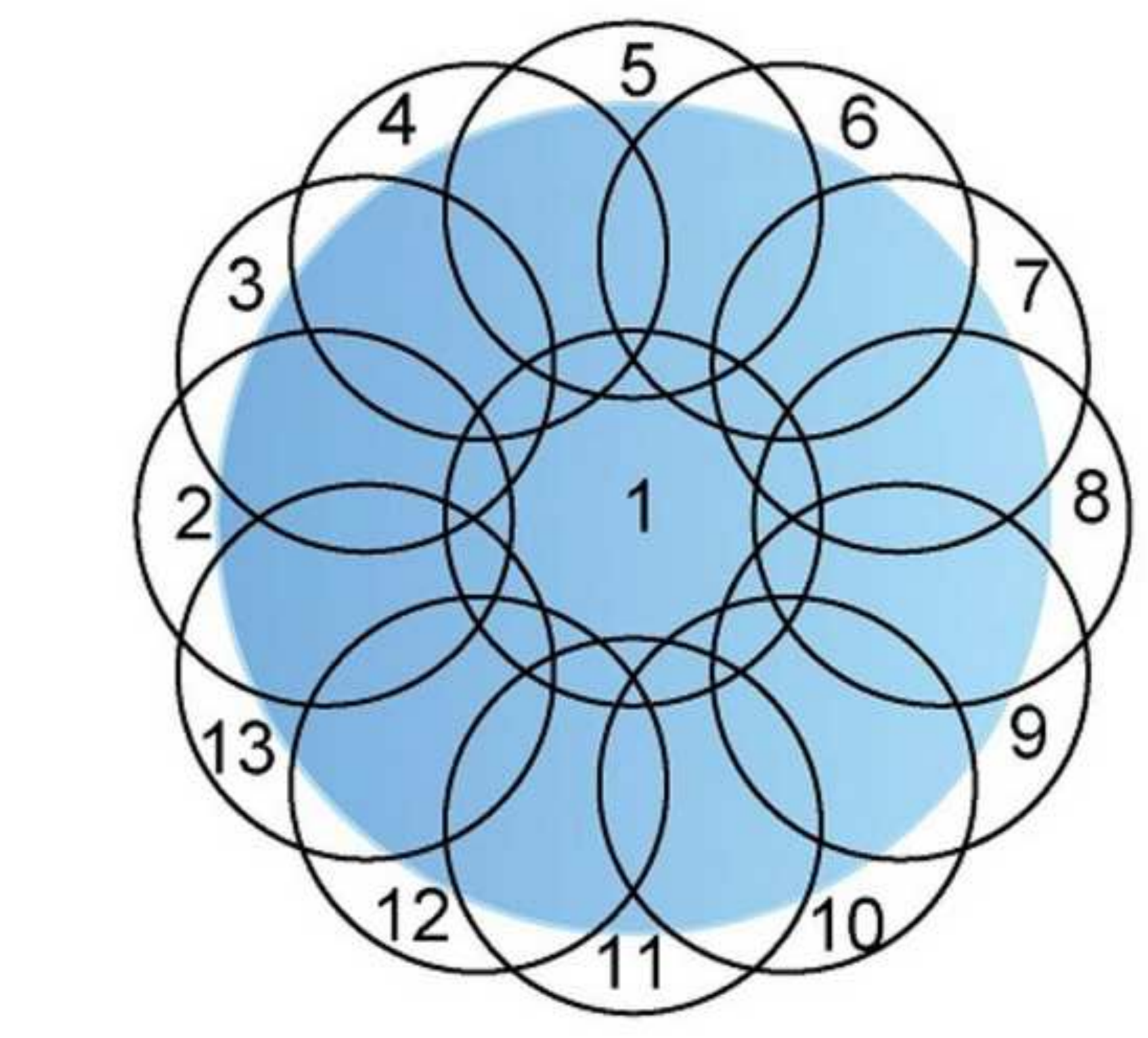




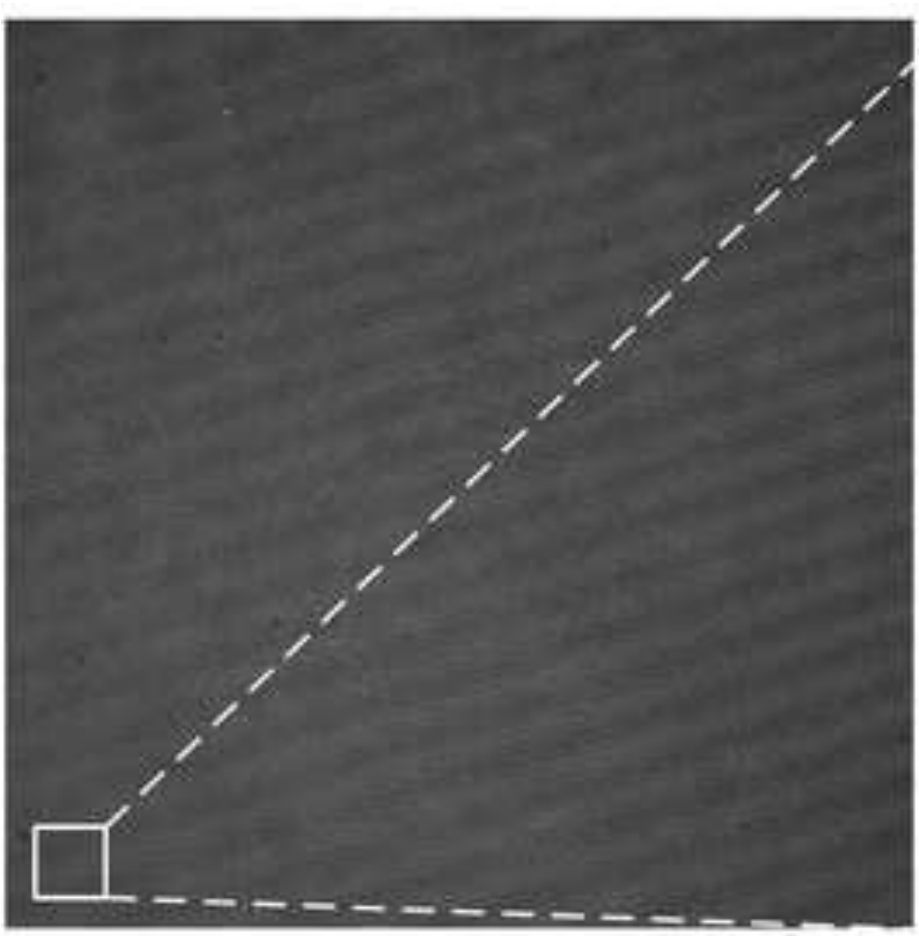

(a)

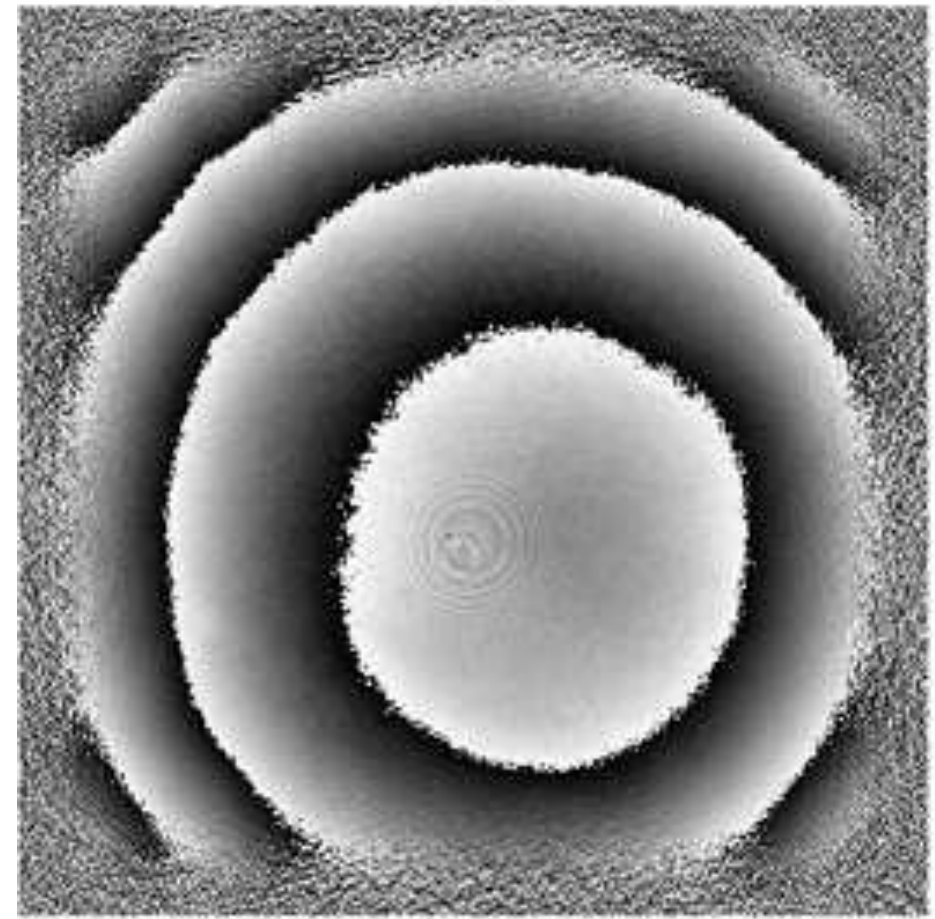

(c)

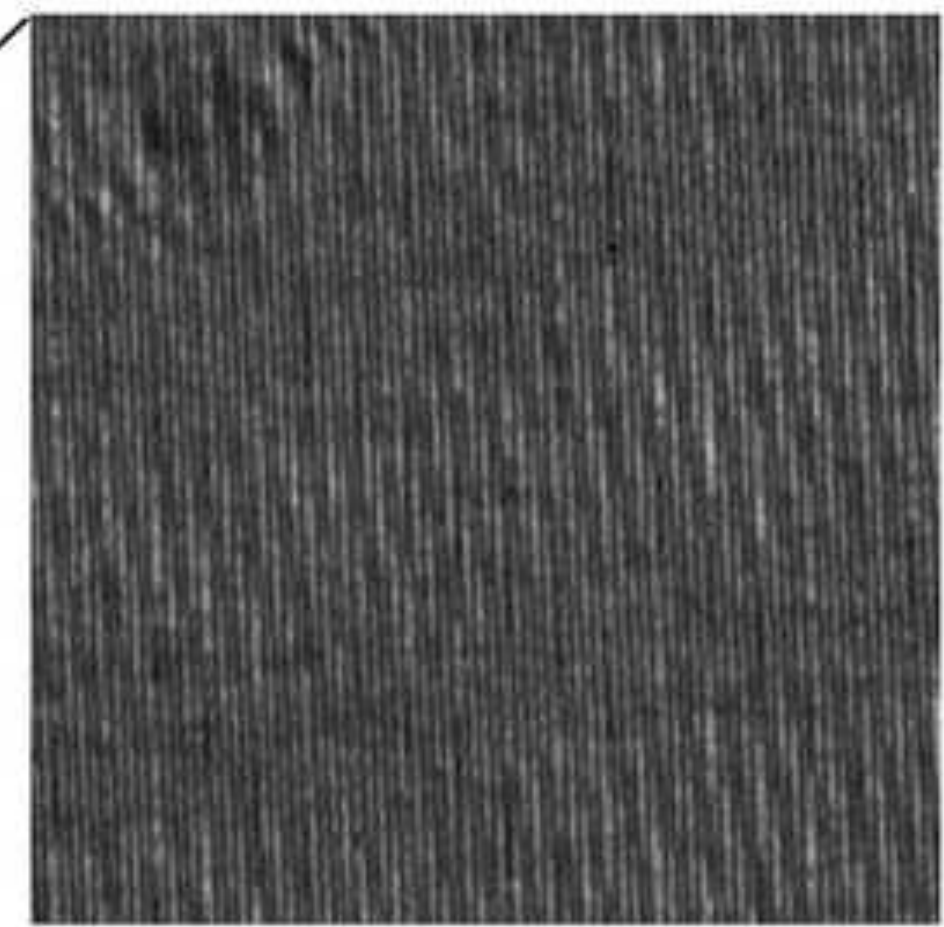

(b)

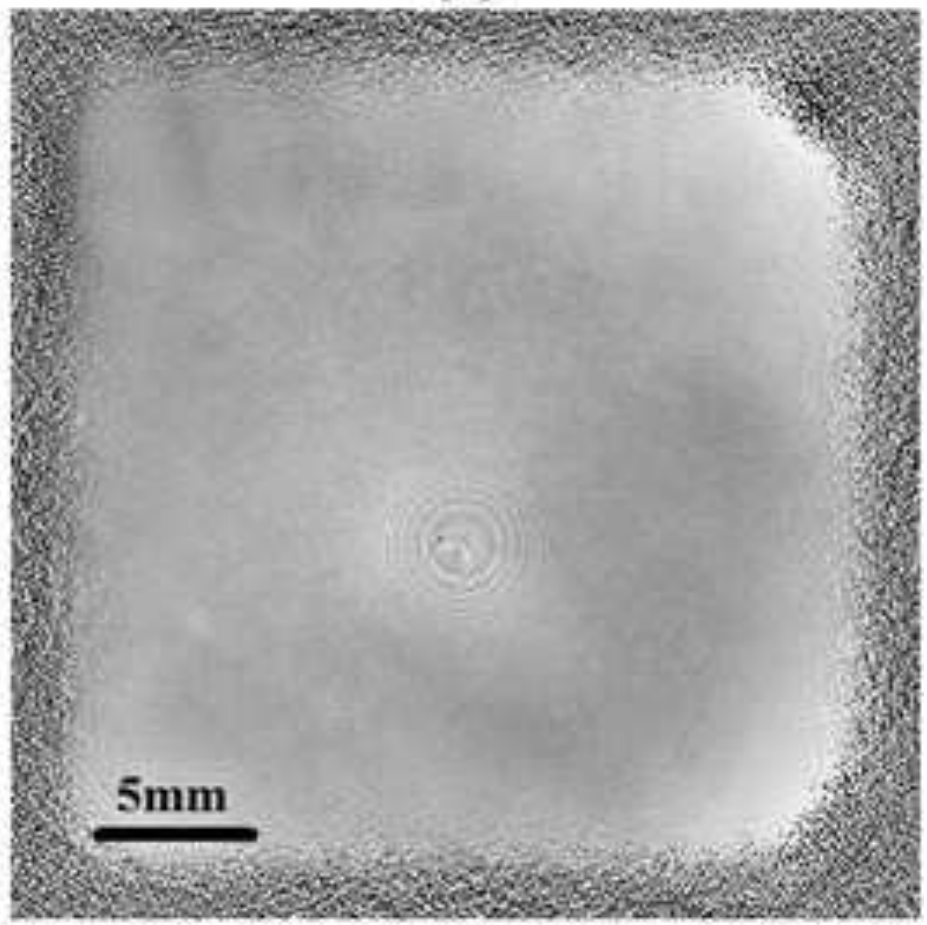

(d) 


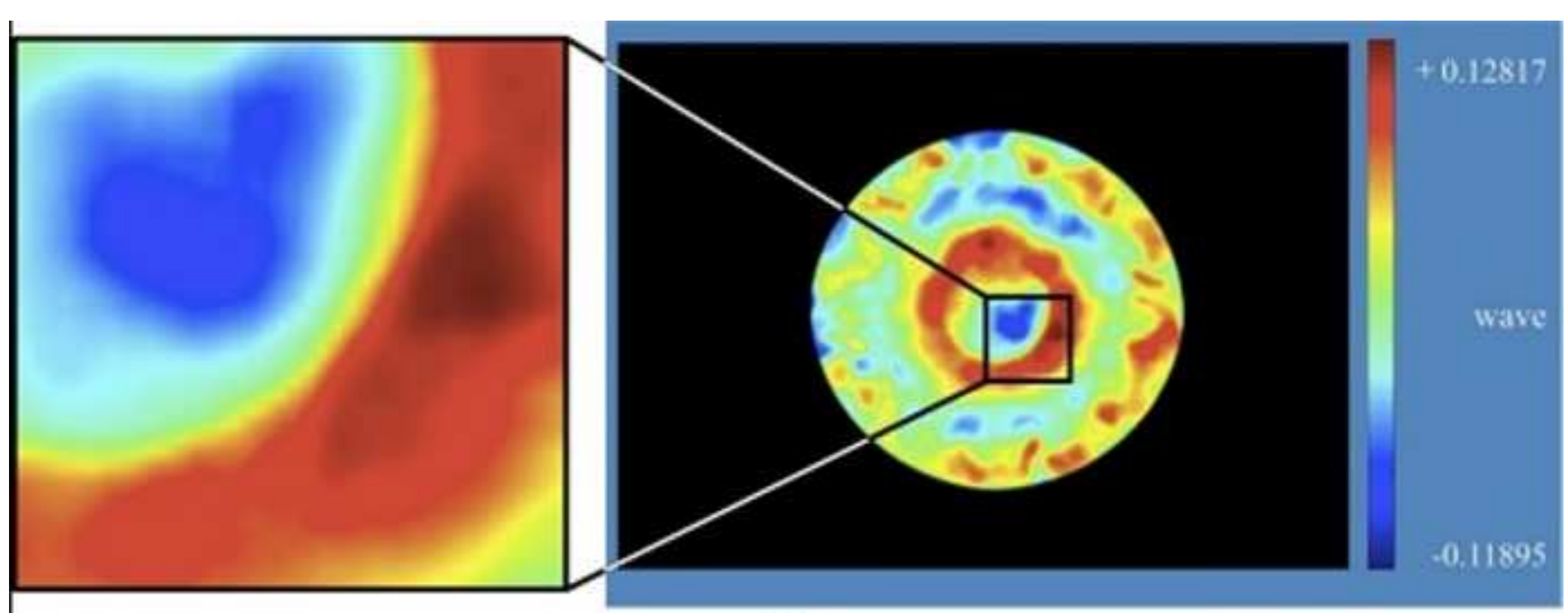

(a)

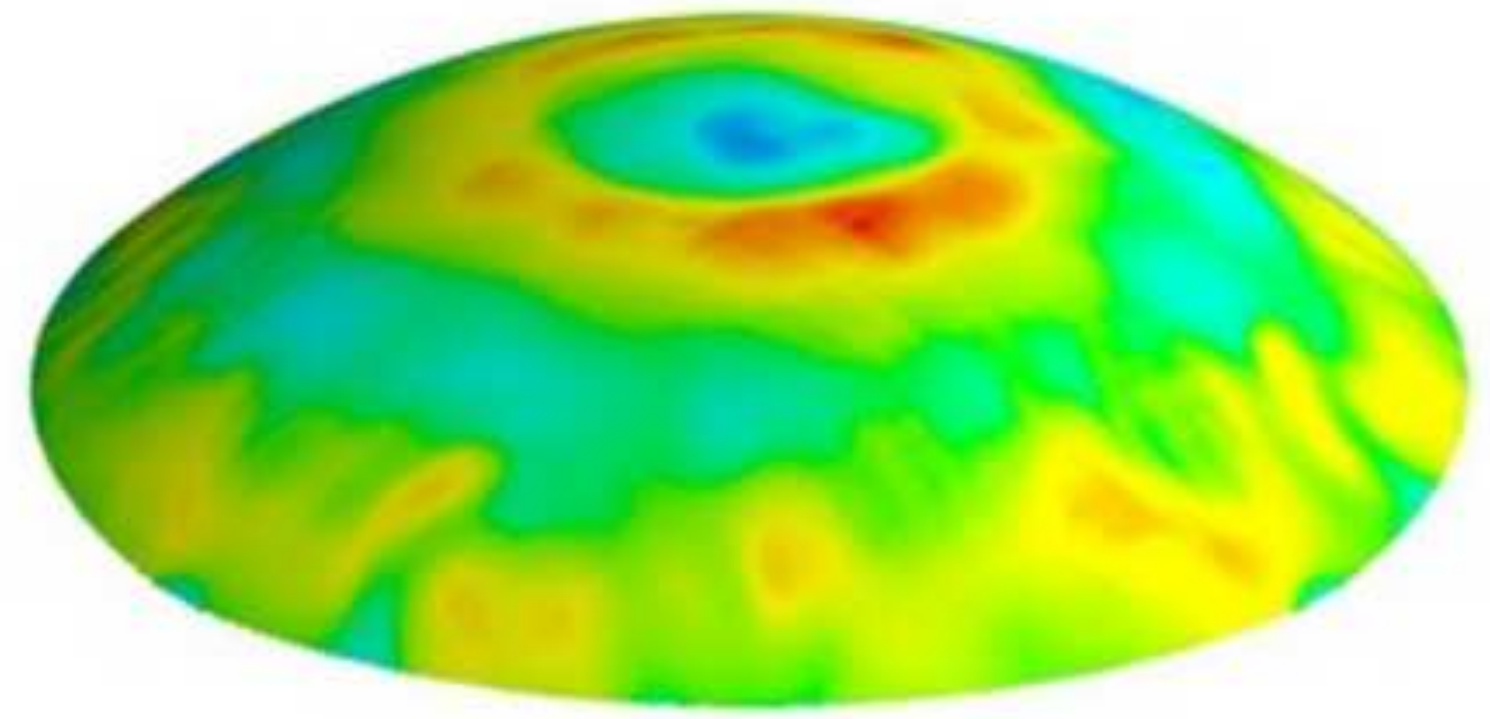

(b) 


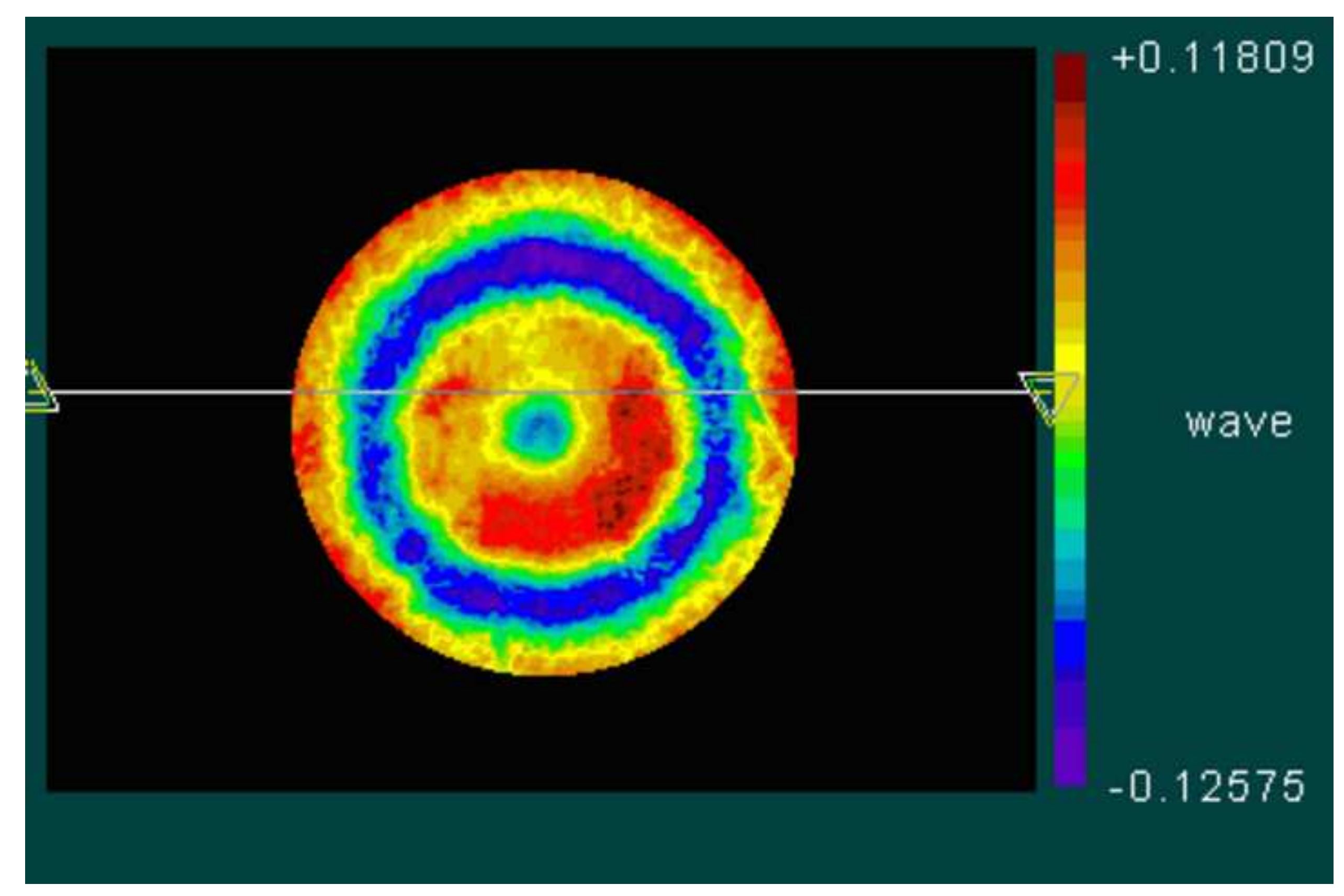

$+0.11809$

wave

$-0.12575$ 

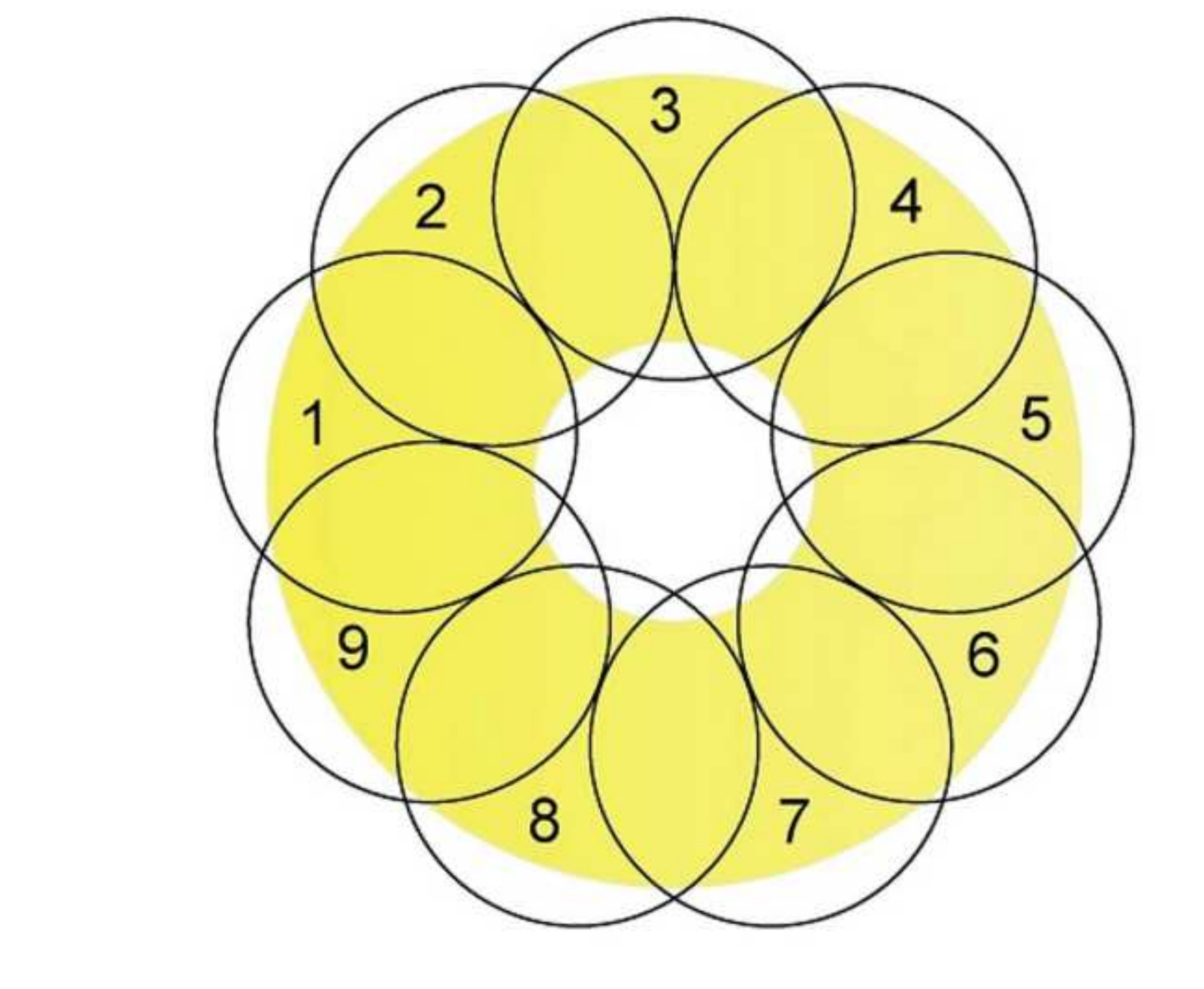
Figure.1C

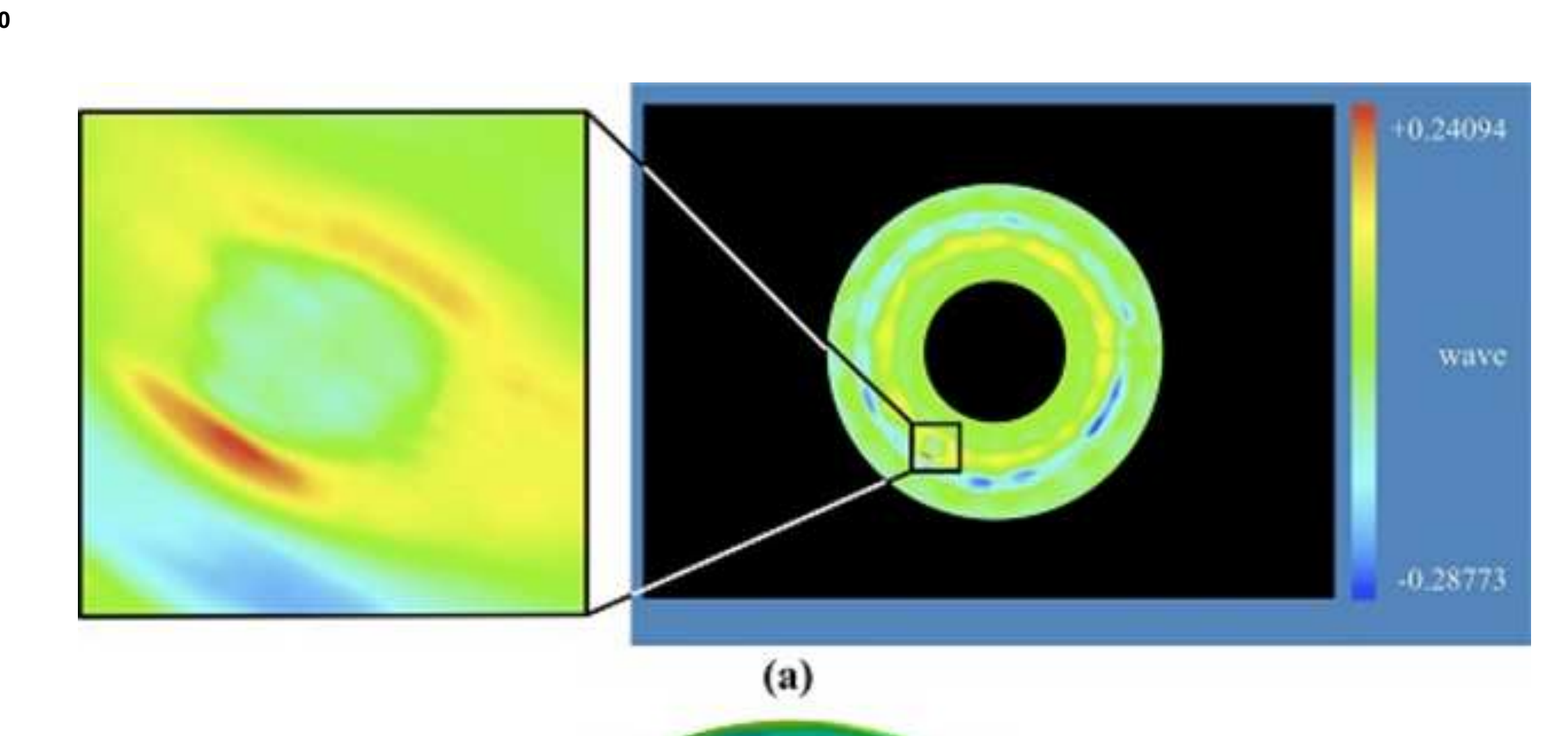

(a)

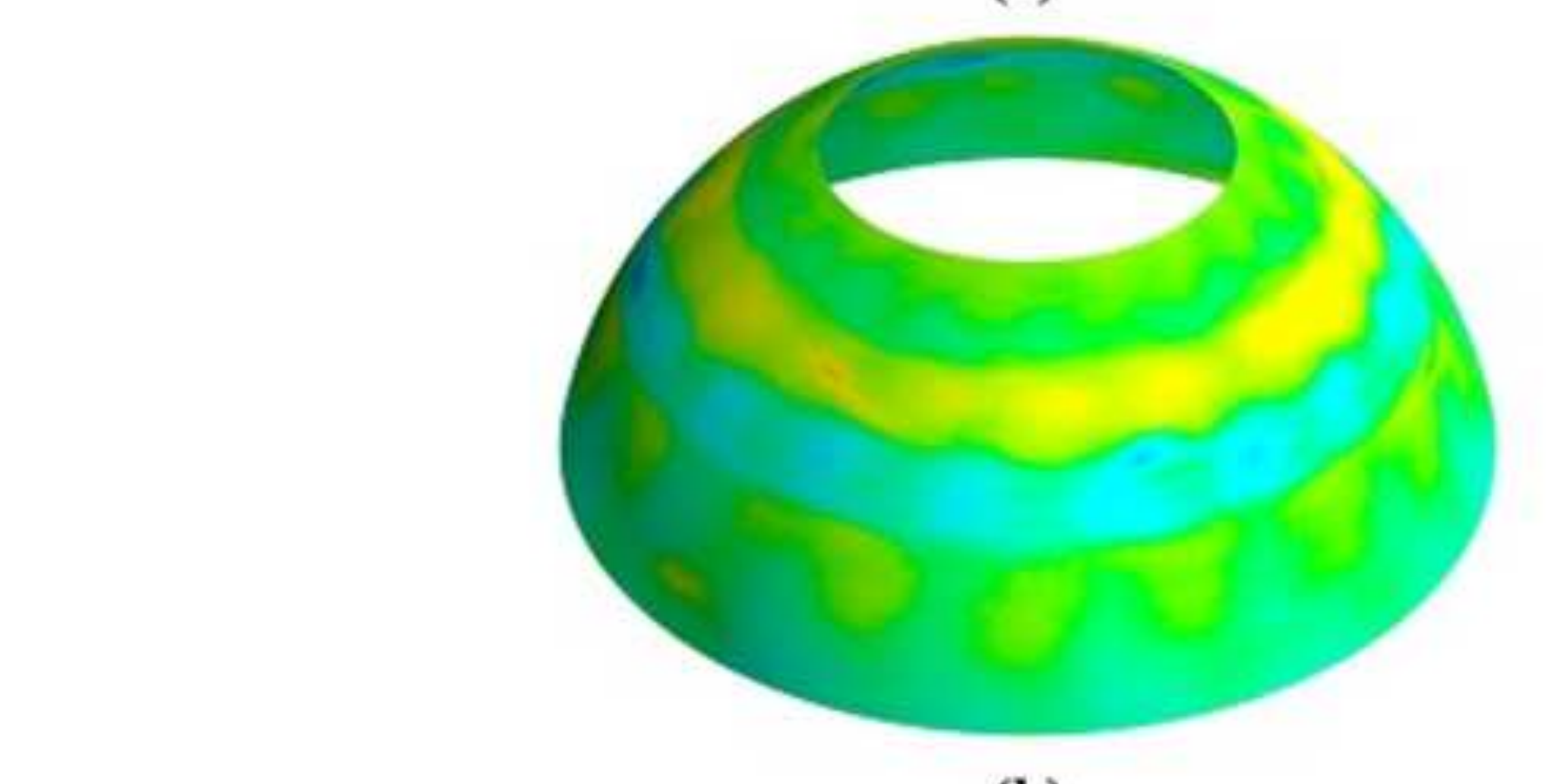

(b)

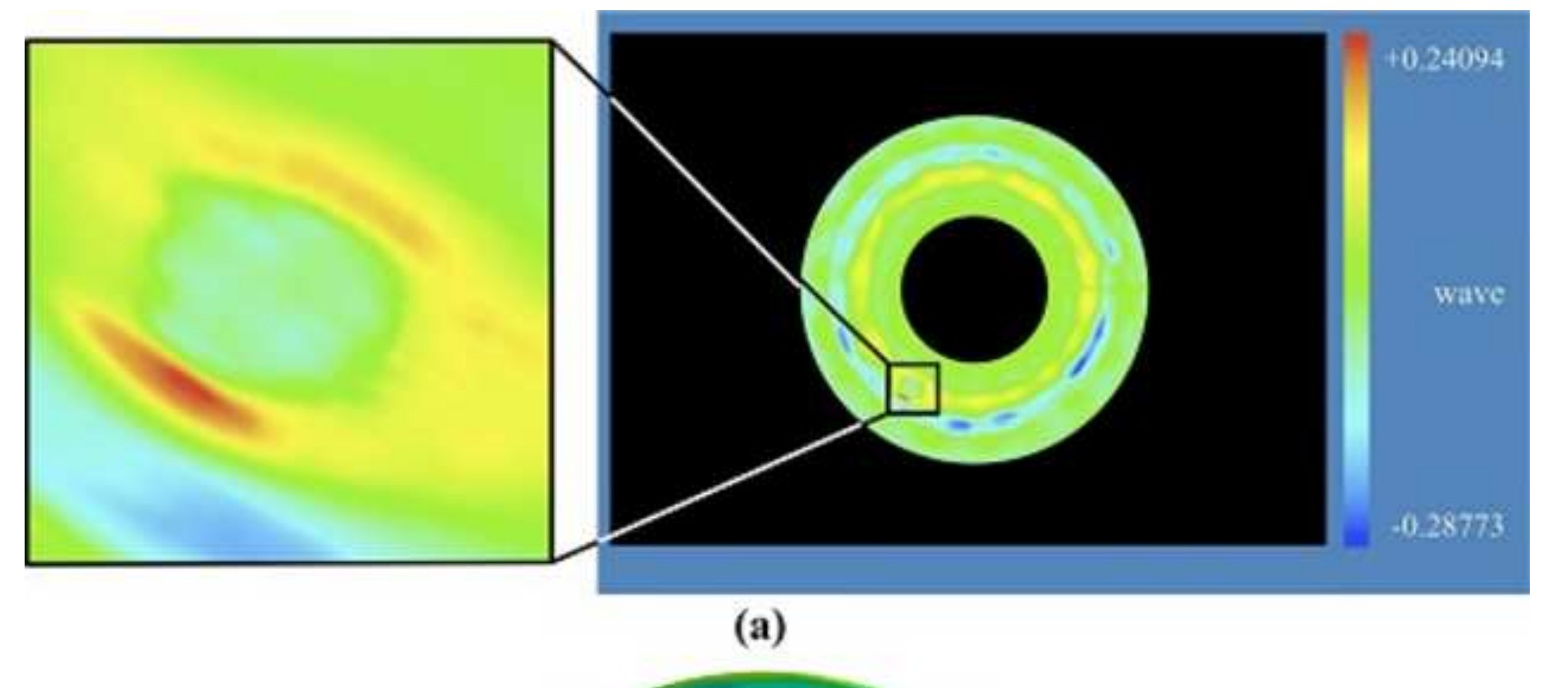

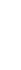

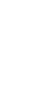

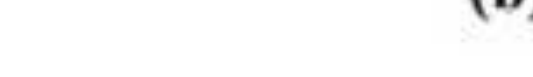
(b)

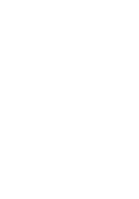
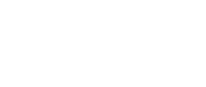